\title{
FERRAMENTA DE ENSINO-APRENDIZAGEM PARA A ESTIMATIVA DO PESO MOLECULAR MÉDIO DE UMA FONTE DE LIPÍDEOS ATRAVÉS DE REAÇÕES DE ESTERIFICAÇÃO
}

\author{
A Teaching-learning tool for estimating the average molecular weight of a \\ source of lipids through esterification reactions
}

\begin{abstract}
Tiago dos Santos Gonçalves ${ }^{1,2}$; Carolina Elisa Demaman Oro ${ }^{1}$; Luciana Dornelles Venquiaturo'; Rogério Marcos Dallago ${ }^{1}$; Marcelo Luis Mignoni ${ }^{1, *}$
\end{abstract}

\footnotetext{
${ }^{1}$ Departamento de Engenharia de Alimentos e Engenharia Química - Universidade Regional Integrada do Alto Uruguai e das Missões (URI), Erechim, RS,

${ }^{2}$ Departamento de Engenharia de Alimentos, IFC - Instituto Federal Catarinense, Concórdia, SC.

*E-mail:mignoni@uricer.edu.br
}

Data do recebimento: 04/03/2021 - Data do aceite: 08/07/2021

RESUMO: No presente estudo, foram abordados os cálculos de esterificação entre um ácido e um álcool, utilizando como molécula modelo o ácido graxo e o glicerol, respectivamente, através do uso de uma planilha de ensino-aprendizagem. O objetivo proposto foi apresentar as fórmulas necessárias para a construção de uma planilha utilizando os softwares como Microsoft Excel ou LibreOffice Calc relacionando dados facilmente mensuráveis de uma fonte de gordura para a obtenção peso molecular médio. Os cálculos apresentados de esterificação foram realizados utilizando dados provenientes da literatura de análises cromatográficas dos ácidos graxos presentes em amostras de óleo de algodão, soja, milho, coco e toucinho. Como resposta, os valores obtidos validaram as fórmulas propostas, mostrando o balanço de massa do sistema (entradas e saídas) e a geração dos produtos na reação. A planilha pode ser facilmente empregada em sala de aula de forma presencial ou online como ferramenta de estudo.

Palavras-chave: Esterificação. Balanço de massa. Peso molecular. 
ABSTRACT: In the present study, the esterification calculations between an acid and an alcohol were presented, using fatty acid and glycerol as the model molecule, respectively, through the use of a teaching-learning spreadsheet. The objective was to present the necessary formulae for the construction of a spreadsheet using software such as Microsoft Excel or LibreOffice Calc, relating easily measurable data from a fat source to obtain average molecular weight. The calculations of esterification presented were performed using data from the literature of chromatographic analyzes of fatty acids present in samples of cottonseed oil, soybean, corn, coconut and bacon. In response, the values obtained validated the proposed formulae, showing the mass balance of the system (inputs and outputs) and the generation of products in the reaction. The spreadsheet can be easily used in the classroom, face-to-face or online, as a study tool.

Keywords: Esterification. Mass balance. Molecular weight.

\section{Introdução}

A relação mais importante em uma reação química é a relação mássica entre os reagentes, a fim de conhecer o reagente limitante, e até mesmo realizar o deslocamento de um potencial equilíbrio químico. Compostos simples como água, minerais e até mesmo combustíveis apresentam facilmente a sua massa molecular. Contudo, fontes de lipídios para a produção de biodiesel são uma mistura mais complexa de ácidos graxos e glicerol (DEMAMAN ORO et al., 2019)using relatively simple physicochemical processes and easy industrial implementation. For this purpose, Total Organic Carbon (TOC, gerando necessidade de análises mais robustas e caras para obter a sua composição. Como ferramenta de ensino-aprendizagem, será abordada a utilização de estequiometria de reação, análise e composição de gorduras, além do balanço de massa para a estimativa do peso molecular médio de uma fonte de lipídeos através de reações de esterificação.

Para tanto, serão mostrados os passos necessários para a construção, assim como os resultados obtidos a partir de uma planilha eletrônica empregada como ferramenta de ensino-aprendizagem, a qual pode ser desenvolvida utilizando softwares como Microsoft Excel ou LibreOffice Calc, ampliando assim o horizonte de aprendizagem e complementando esta atividade de importância para disciplinas de Química Geral, Balanço de Massa, Análise de Alimentos e práticas de laboratórios para a Engenharia (CABEZA et al., 2016; SIMÓN-HERRERO et al., 2019) this program could simulate the dynamic concentration profiles inside the column, being a useful teaching tool. The model comprises non-stationary mass balances for recovered compounds in both phases (solid and supercritical $\mathrm{CO} 2$.

O Brasil é um dos maiores produtores e consumidores de biocombustíveis do mundo devido à sua alta produção e disponibilidade de matérias-primas (CREMONEZ et al., 2015). O biodiesel é um combustível obtido a partir de matérias-primas vegetais ou animais. As matérias-primas vegetais são derivadas de óleos vegetais, tais como soja, mamona, colza (canola), palma, girassol e amendoim, entre outros. As matérias-primas de origem animal são obtidas do sebo bovino, suíno e de aves. Incluem-se entre as alterna- 
tivas de matérias-primas os óleos utilizados em frituras (cocção) (GAUTO; ROSA, 2011).

As empresas em operação adotam a tecnologia denominada transesterificação, com predominância da rota tecnológica metílica em comparação com a etílica, no Brasil. A rota etílica não separa bem os produtos obtidos e ainda requer aperfeiçoamento tecnológico. Dessa forma, no processo de transesterificação para a produção de biodiesel, o metanol reage com o triglicerídeo (óleo/gordura) na presença de um catalisador básico ou ácido para formar ésteres metílicos de ácidos graxos. As reações de transesterificação dos óleos vegetais, além de produzir o biodiesel, geram em torno de $10 \%$ de glicerina, que vem se destacando como um atraente insumo químico oriundo de biomassa e, portanto, renovável (MOTA; PINTO, 2017).

A estequiometria geralmente consiste em 3 mols de álcool para $1 \mathrm{~mol}$ de triglicerídeo, mas na prática um excesso de álcool é utilizado para aumentar o rendimento na formação de ésteres (ENCIMAR et al., 2002). No presente estudo, será abordada a esterificação entre um ácido (representado pelo ácido graxo) e um álcool (representado pelo glicerol).

Diante do exposto, o objetivo do presente estudo é apresentar uma metodologia para construção de uma planilha de ensino-aprendizagem relacionando dados facilmente mensuráveis de uma fonte de gordura para obtenção de seu peso molecular médio, com a finalidade de ser empregada para fins de balanceamento de reações, como, por exemplo, na produção de biodiesel.

\section{Metodologia}

A Tabela I apresenta uma relação de tipos de ácidos graxos com seu peso molecular,

Tabela I - Ácidos graxos e seus respectivos pesos moleculares (FENNEMA; DAMODARAN; PARKIN, 2010)

\begin{tabular}{cccc}
\hline $\begin{array}{c}\mathbf{N}^{\mathbf{0}} \mathbf{d e} \\
\text { carbonos }\end{array}$ & Tipo de ácido graxo & Ácido graxo & $\begin{array}{c}\text { Peso } \\
\text { molecular } \\
\text { mol) }\end{array}$ \\
\hline 4 & SAT & 88,1051 \\
5 & SAT & Butírico & 102,1300 \\
6 & SAT & Valérico & 116,1583 \\
8 & SAT & Capróico & 144,2100 \\
10 & SAT & Caprílico ou Octanóico & 172,2600 \\
12 & SAT & Cáprico ou Decanóico & 200,3178 \\
14 & SAT & Láurico ou Duodecanóico & 228,3709 \\
14 & INSAT & Mirístico ou Tertradecanóico & 226,2600 \\
16 & SAT & Miristoleico ou 9-Tetradecenóico & 256,4000 \\
16 & INSAT & Palmítico ou Hexadecanóico & 254,4082 \\
18 & SAT & Palmitoléico ou Hexadecenóico & 284,4800 \\
18 & INSAT & Esteárico ou Octadecanóico & 282,4600 \\
18 & INSAT & Oléico ou Octadecenóico & 280,4455 \\
18 & INSAT & Linoléico ou Octadecadienóico & 278,4300 \\
20 & SAT & Linolênico ou Octadecatrienóico & 312,5300 \\
24 & SAT & Araquídico ou Eicosanóico & 368,6300 \\
30 & SAT & Lignocérico ou Tetracosanóico & 452,7900 \\
\hline
\end{tabular}


que servirá de base para estimar o número de mols dos mesmos, em uma fonte de lipídeos. Pode-se observar que a Tabela I apresenta o tipo de ácido graxo em relação ao número de carbonos e também pela saturação, apresendo os ácidos graxos saturados (SAT) e insaturados (INSAT).

Através de uma análise cromatográfica de fontes de óleos e graxas, tem-se a composição da gordura em termos de ácidos graxos. Com esta informação, pode-se avaliar qual o número de mols de ácidos presentes nesta amostra de gordura, utilizando-se uma base de cálculo qualquer, conforme descrito através da Equação (1). Os resultados das análises cromatográficas podem ser facilmente encontrados na literatura para fins de cálculo.

$$
n_{\text {TOTAL }}=\sum_{i=1}^{N}\left[\frac{M_{B A S E} \times x_{i}}{P M_{i}}\right]
$$

Em que: é o número total de ácido graxos na amostra de gordura, é a base de cálculo para balanço de massa (g), é a fração mássica de um ácido graxo na amostra de gordura, e é a massa molecular do ácido graxo $(\mathrm{g} / \mathrm{mol})$.

Como a gordura é formada pela reação de ácidos graxos e glicerol, também denominado de triglicerídeos, a massa total da gordura deve estar inclusa com a massa de glicerol em uma proporção molar de 3 mols de ácidos graxos para $1 \mathrm{~mol}$ de glicerol. A Equação (2) representa o número de mols de glicerol envolvidos na construção da amostra de gordura.

$$
n_{\text {GLICEROL }}=\frac{n_{\text {TOTAL }}}{3}
$$

Em que: é o número de mols de glicerol envolvidos na produção da gordura, e é o número total de ácido graxos na amostra de gordura obtido pela Equação (1).
Assim, a massa de glicerol que será adicionada para a produção de lipídeos será apresentada pela Equação (3).

$$
m_{\text {GLICEROL }}=92,0776 \times n_{\text {GLICEROL }}
$$

Em que: é a massa de glicerol (g) adicionada ao sistema para a produção de lipídeos, é o número de mols de glicerol envolvidos na produção da gordura obtido pela Equação (2), e o fator 92,0776 é a massa molar de glicerol $(\mathrm{g} / \mathrm{mol})$.

A reação de esterificação é a reação entre um ácido (representado pelo ácido graxo) e um álcool (representado pelo glicerol). Dessa forma, através da estequiometria descrita pela Equação (4), percebe-se que para cada $1 \mathrm{~mol}$ de ácido graxo como reagente no sistema, ocorre a saída de $1 \mathrm{~mol}$ de água resultante da reação de esterificação. Portanto, a massa que sai do sistema e deve ser contabilizada no balanço de massa.

3Ácidograxo + 1glicerol $\rightarrow 1$ triglicerídio +3 Água

Diante desta informação, podemos afirmar que o número de mols de água que sai do sistema reacional é igual ao número de mols de ácido graxo que entra no sistema, conforme descrito através da Equação (1). Assim, a massa de água que sai do sistema é apresentada conforme Equação (5).

$$
m_{\text {ÁGUA }}=18,01528 \times n_{\text {TOTAL }}
$$

Em que: é a massa de água (g) que sai do sistema para a produção de lipídeos, é o número de mols de ácido graxo envolvidos na produção da gordura obtido pela Equação (1), e o fator 18,01528 é a massa molar da água $(\mathrm{g} / \mathrm{mol})$. 
Portanto, a massa total da amostra de gordura pode ser calculada através da Equação (6).

$m_{\text {AMOSTRA }}=M_{B A S E}+m_{\text {GLICEROL }}-m_{\text {ÁGUA }}$

Em que: é a massa total (g) da amostra de gordura que foi analisada, é a massa ( $\mathrm{g}$ ) da base de cálculo para avaliação de número de mols de ácido graxo para a Equação (1), é a massa de glicerol (g) adicionada ao sistema para a produção de lipídeos obtida pela Equação (3), e é a massa de água (g) que sai do sistema para a produção de lipídeos obtida pela Equação (5).

De posse do resultado da Equação (6), pode-se estimar o peso molecular médio da amostra de gordura $(\mathrm{g} / \mathrm{mol})$ através da Equação (7), pois através da estequiometria da formação de gorduras (Equação 4), tem-se que o número de mols de glicerol é o mesmo número de mols da gordura que foi analisada.

$$
P M_{\text {GORDURA }}=\frac{m_{\text {AMOSTRA }}}{n_{\text {GLICEROL }}}
$$

Em que: é a massa total (g) da amostra de gordura que foi analisada obtida pela Equação (6), e é o número de mols de glicerol envolvidos na produção da gordura obtido pela Equação (2).

Os cálculos do presente estudo foram realizados utilizando os dados provenientes de análises cromatográficas dos ácidos graxos presentes em amostras de óleo de algodão, soja, milho, coco e toucinho (Tabela II). A partir das Equações apresentadas no presente estudo, uma planilha de ensino-aprendizagem foi facilmente montada e pode ser implementada em sala de aula utilizando os softwares como Microsoft Excel ou LibreOffice Calc.

\section{Resultados e Discussão}

A Figura 1 apresenta os resultados obtidos para o total de mol de ácidos graxos, glicerol

Tabela II - Ácidos graxos presentes em diferentes fontes de gordura vegetal e animal (FONSECA; GUTIERREZ, 1974; GONDIM-TOMAZ et al., 2016)

\begin{tabular}{cccccc}
\hline Ácido graxo & $\begin{array}{c}\text { Óleo de } \\
\text { algodão } \\
(\mathbf{g} / \mathbf{1 0 0 g})\end{array}$ & $\begin{array}{c}\text { Óleo } \\
\mathbf{d e} \mathbf{s o j a} \\
(\mathbf{g} / \mathbf{1 0 0 g})\end{array}$ & $\begin{array}{c}\text { Óleo de } \\
\text { milho } \\
(\mathbf{g} / \mathbf{1 0 0 g})\end{array}$ & $\begin{array}{c}\text { Óleo } \\
\mathbf{d e ~ c o c o} \\
\mathbf{( g / 1 0 0 g})\end{array}$ & $\begin{array}{c}\text { Toucinho } \\
(\mathbf{g} / \mathbf{1 0 0 g})\end{array}$ \\
\hline Caprílico ou Octanóico & - & - & - & 8,00 & - \\
Cáprico ou Decanóico & - & - & - & 7,00 & - \\
Láurico ou Duodecanóico & - & - & - & 48,00 & - \\
Mirístico ou Tertradecanóico & 0,60 & - & - & 17,50 & 1,00 \\
Miristoleico ou 9-Tetradecenóico & - & - & - & - & 0,30 \\
Palmítico ou Hexadecanóico & 22,90 & 8,30 & 7,50 & 8,80 & 28,00 \\
Palmitoléico ou Hexadecenóico & - & - & - & - & 3,00 \\
Esteárico ou Octadecanóico & 2,20 & 5,40 & 3,50 & 2,20 & 13,00 \\
Oléico ou Octadecenóico & 24,70 & 24,90 & 46,30 & 6,00 & 46,00 \\
Linoléico ou Octadecadienóico & 49,60 & 52,40 & 42,20 & 2,50 & 6,00 \\
Linolênico ou Octadecatrienóico & - & 7,90 & - & - & 0,70 \\
Araquídico ou Eicosanóico & - & 0,90 & 0,50 & - & 2,00 \\
Lignocérico ou Tetracosanóico & - & 0,20 & - & - & - \\
\hline
\end{tabular}


e água das amostras após os cálculos realizados na ferramenta de ensino. É possível observar que o número total de mols de ácidos graxos é igual ao número total de mols de água, e também é o triplo do valor do total de mols de glicerol. Isso valida novamente que o número de mols de água que sai do sistema reacional é igual ao número de mols de ácido graxo que entra no sistema para todas as fontes de gordura em estudo.

Figura 1 - Total de mols no sistema para as diferentes fontes de gordura vegetal e animal

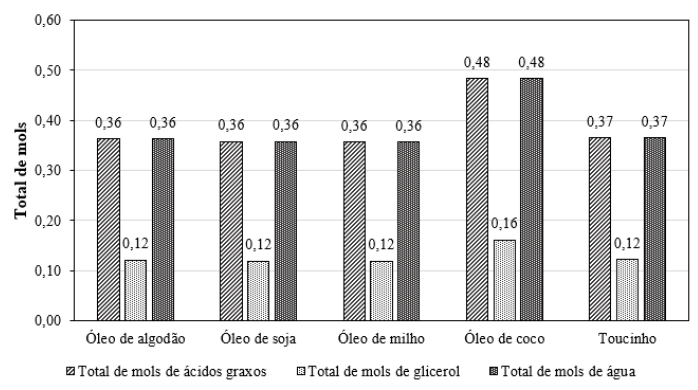

A Figura 2 apresenta a massa de água (g) que sai do sistema para a produção de lipídeos e a massa de glicerol (g) adicionada ao sistema para a produção de lipídeos, conforme apresentado na Equação 4. É possível observar que a relação entre a massa de glicerol e água é praticamente a mesma em todas as amostras em estudo, sendo a quantidade de massa de água correspondente a aproximadamente $58 \%$ do valor de massa de glicerol. Com base nesses resultados foi possível calcular a massa (g) da amostra a ser analisada para obtenção do respectivo peso molecular, e os resultados estão apresentados na Tabela III.

Com base nos resultados apresentados na Tabela III, é possível observar resultados semelhantes para a massa ( $g$ ) de amostra em análise. Entretanto, diferentes pesos moleculares médios são encontrados para as amostras. A amostra que apresentou maior peso molecular médio para os dados de entrada, foi o óleo de milho $(877,08 \mathrm{~g} / \mathrm{mol})$. Em contraste, o óleo de coco apresentou um peso molecular médio de 657,14 g/mol.

Figura 2 - Valores calculados de massa (g) de água e glicerol, representando a saída e entrada de massa do sistema, respectivamente

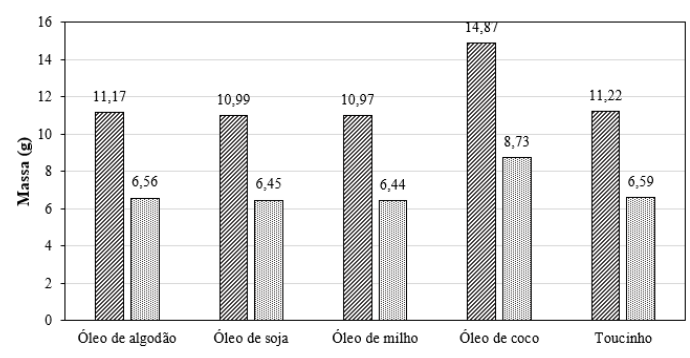

Tabela III - Massa de amostra em análise e seu respectivo peso molecular médio (PM)

\begin{tabular}{ccc}
\hline Amostra & $\begin{array}{c}\text { Massa da } \\
\text { amostra em } \\
\text { análise (g) }\end{array}$ & $\begin{array}{c}\text { Peso molecular } \\
\text { médio da fonte } \\
\text { de gordura (g/ } \\
\text { mol) }\end{array}$ \\
\hline Óleo de algodão & 104,61 & 862,25 \\
Óleo de soja & 104,54 & 875,67 \\
Óleo de milho & 104,53 & 877,08 \\
Óleo de coco & 106,14 & 657,14 \\
Toucinho & 104,63 & 858,69 \\
\hline
\end{tabular}

Além dos resultados apresentados, a planilha pode mostrar, rapidamente, novos resultados ao ter seus dados de entrada modificados, avaliando gorduras de diferentes fontes e genótipos. Isso permite a comparação e estudo de diferentes óleos e gorduras, os quais podem apresentar diferente composição em função da época do ano, solo, clima, e outros fatores. A Figura 3 apresenta uma visão geral da planilha montada no presente estudo.

É muito importante o uso de ferramentas, como a apresentada pelo presente estudo, em sala de aula. Os professores devem incorporar 
o uso desse tipo de ferramenta no ensino presencial ou online, com o intuito de auxiliar na construção de abordagens de ensino construcionistas e instrucionistas (PEREIRA; DE BARROS, 2020). Além de contribuir com a análise do estudo proposto em si, os alunos aprendem a usar diferentes softwares, os quais são um diferencial para o estágio e mercado de trabalho em diferentes áreas, principalmente na modelagem e simulação de processos (AGUDELO-RODRÍGUEZ;
MONCAYO-MARTÍNEZ; CASTELLANOS, 2020; OZTURK; TAKHAR, 2018).

\section{Conclusão}

O presente estudo apresentou com sucesso a construção de uma planilha de ensino-aprendizagem relacionando dados facilmente mensuráveis de uma fonte de gordura com seu peso molecular médio. Como exemplo,

Figura 3 - Visão geral da planilha de ensino-aprendizagem utilizada no presente estudo.

\begin{tabular}{|c|c|c|c|c|c|c|c|}
\hline \multirow{2}{*}{\multicolumn{2}{|c|}{$\begin{array}{l}\text { Base de Cálculo }(g) \\
100\end{array}$}} & & \multicolumn{5}{|c|}{ PM Médio da fonte de gordura (g/mol) } \\
\hline & & & \multicolumn{5}{|c|}{ Equação (07) } \\
\hline & & & Óleo de algodão & Óleo de soja & Óleo de milho & Óleo de coco & Toucinho \\
\hline & & & 862,2489 & 875,6729 & 877,0845 & 657,1361 & 858,6870 \\
\hline & & & \multicolumn{5}{|c|}{ ( $\mathrm{g} / 100 \mathrm{~g}$ de óleo) - Análise de Cromatografia } \\
\hline $\mathrm{N}^{\circ}$ de & Tipo Ácido & & 100 & 100 & 100 & 100 & 100 \\
\hline Carbono & Graxo & Ácido & Óleo de algodão & Óleo de soja & óleo de milho & Óleo de coco & Toucinho \\
\hline 4 & SAT & Butírico & & & & & \\
\hline 5 & SAT & Valérico & & & & & \\
\hline 6 & SAT & Capróico & & & & & \\
\hline 8 & SAT & $\begin{array}{l}\text { Caprílico } \\
\text { Octanóico }\end{array}$ & & & & 8,00 & \\
\hline 10 & SAT & $\begin{array}{l}\text { Cáprico } \\
\text { Decanóico }\end{array}$ & & & & 7,00 & \\
\hline 12 & SAT & $\begin{array}{c}\text { Láurico } \\
\text { Duodecanóico }\end{array}$ & & & & 48,00 & \\
\hline 14 & SAT & $\begin{array}{c}\text { Mirístico } \\
\text { Tertradecanóico }\end{array}$ & 0,60 & & & 17,50 & 1,00 \\
\hline 14 & INSAT & $\begin{array}{l}\text { Miristoleico } \\
\text { 9-Tetradecenóico }\end{array}$ & & & & & 0,30 \\
\hline 16 & SAT & $\begin{array}{c}\text { Palmítico } \\
\text { Hexadecanóico }\end{array}$ & 22,90 & 8,30 & 7,50 & 8,80 & 28,00 \\
\hline 16 & INSAT & $\begin{array}{l}\text { Palmitoléico } \\
\text { Hexadecenóico }\end{array}$ & & & & & 3,00 \\
\hline 18 & SAT & $\begin{array}{c}\text { Esteárico } \\
\text { Octadecanóico }\end{array}$ & 2,20 & 5,40 & 3,50 & 2,20 & 13,00 \\
\hline
\end{tabular}

Número de Mols de cada ácido graxo - função da fração mássica

\begin{tabular}{c:cc:cc:c}
\hline PM (g/mol) & Óleo de algodão & Óleo de soja & Óleo de milho & Óleo de coco & Toucinho \\
\hdashline 88,1051 & $0,000000 \mathrm{E}+00$ & $0,000000 \mathrm{E}+00$ & $0,000000 \mathrm{E}+00$ & $0,000000 \mathrm{E}+00$ & $0,000000 \mathrm{E}+00$ \\
\hdashline $\mathbf{1 0 2 , 1 3 0 0}$ & $0,000000 \mathrm{E}+00$ & $0,000000 \mathrm{E}+00$ & $0,000000 \mathrm{E}+00$ & $0,000000 \mathrm{E}+00$ & $0,000000 \mathrm{E}+00$ \\
\hdashline $\mathbf{1 1 6 , 1 5 8 3}$ & $0,000000 \mathrm{E}+00$ & $0,000000 \mathrm{E}+00$ & $0,000000 \mathrm{E}+00$ & $0,000000 \mathrm{E}+00$ & $0,000000 \mathrm{E}+00$
\end{tabular}

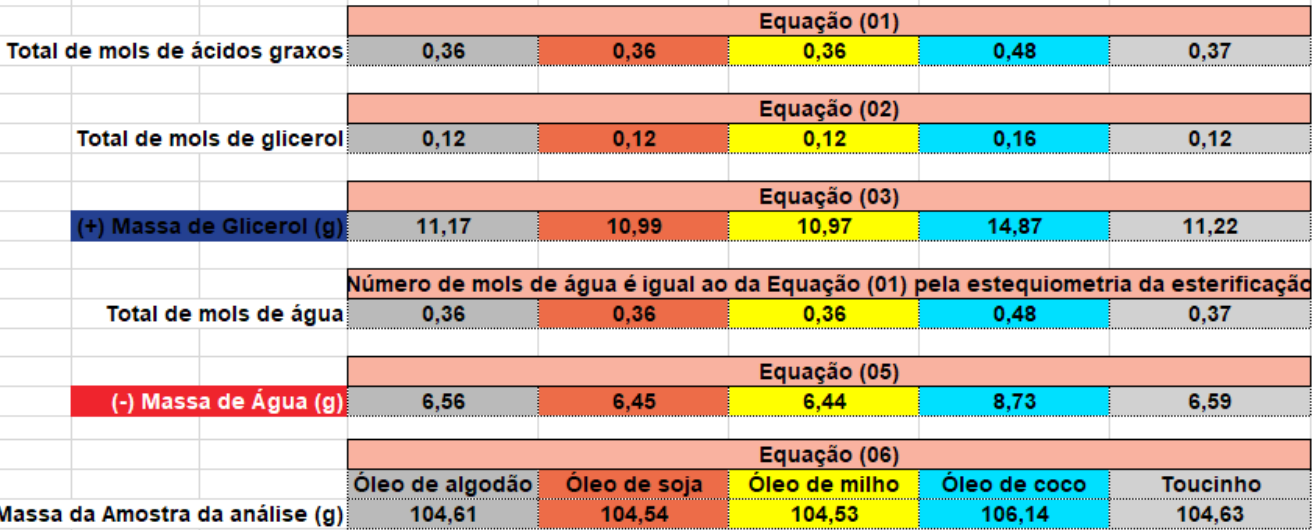


foi apresentado o balanceamento de reações na produção de biodiesel. Como resposta, os valores obtidos validaram as fórmulas propostas, mostrando o balanço de massa do sistema e a geração dos produtos na reação.

\section{REFERÊNCIAS}

AGUDELO-RODRÍGUEZ, G.; MONCAYO-MARTÍNEZ, D.; CASTELLANOS, D. A. Evaluation of a predictive model to configure an active packaging with moisture adsorption for fresh tomato. Food Packaging and Shelf Life, v. 23, n. April 2019, p. 100458, 2020.

CABEZA, A.; SOBRÓN, F.; GARCÍA-SERNA, J.; COCERO, M. J. Simulation of the supercritical $\mathrm{CO} 2$ extraction from natural matrices in packed bed columns: User-friendly simulator tool using Excel. Journal of Supercritical Fluids, v. 116, p. 198-208, 2016.

CREMONEZ, P. A.; FEROLDI, M.; NADALETI, W. C.; DE ROSSI, E.; FEIDEN, A.; DE CAMARGO, M. P.; CREMONEZ, F. E.; KLAJN, F. F. Biodiesel production in Brazil: current scenario and perspectives. Renewable \& Sustainable Energy Reviews, v. 42, p.415-428, 2015.

DEMAMAN ORO, C. E.; BONATO, M.; OLIVEIRA, J. V.; TRES, M. V.; MIGNONI, M. L.; DALLAGO, R. M. A new approach for salts removal from crude glycerin coming from industrial biodiesel production unit. Journal of Environmental Chemical Engineering, v. 7, n. 1, p. 102883, 2019.

ENCIMAR, J. M.; GONZÁLEZ, J. F.; RODRÍGUEZ, J. J.; TEJEDOR, A. Biodiesel Fuels from Vegetable Oils: Transesterification of Cynara cardunculus L. Oils with Ethanol. Energy Fuels, v. 16, n. 2, p.443-450, 2002.

FENNEMA, O. R.; DAMODARAN, S.; PARKIN, K. L. Química de Alimentos de Fennema. 4. ed. - Editora Artmed, 2010.

FONSECA, H.; GUTIERREZ, L. E. Composição em ácidos graxos de óleos vegetais e gorduras animais. Anais da Escola Superior de Agricultura Luiz de Queiroz, v. 31, n. 0, p. 485-490, 1974.

GAUTO, M. A.; ROSA, G. R. Processos e Operações Unitárias da Indústria Química. Rio de Janeiro: Editora Ciência Moderna Ltda., 2011.

GONDIM-TOMAZ, R. M. A.; MAGALHÃES ERISMANN, N. DE; CIA, E.; KONDO, J. I.;

FUZATTO, M. G.; CARVALHO, C. R. L. Teor de óleo e composição de ácidos graxos em sementes de diferentes genótipos de algodoeiro. Brazilian Journal of Food Technology, v. 19, p. 1-8, 2016.

MOTA, C. J. A.; PINTO, B. P. Transformações Catalíticas do Glicerol para Inovação na Indústria Química. Revista Virtual de Química, v. 9, n. 1, p. 135-149, 2017.

OZTURK, O. K.; TAKHAR, P. S. Water transport in starchy foods: Experimental and mathematical aspects. Trends in Food Science and Technology, v. 78, n. May, p. 11-24, 2018.

PEREIRA, M. D.; BARROS, E. A. DE. A educação e a escola em tempos de coronavírus. Scientia Vitae, v. 9, n. 28, p. 1-7, 2020.

SIMÓN-HERRERO, C.; ROMERO, A.; DORADO, F.; GRACIA, I.; VALVERDE, J. L.;

SANCHEZ-SILVA, L. Taylor-made aerogels through a freeze-drying process: economic assessment. Journal of Sol-Gel Science and Technology, v. 89, n. 2, p. 436-447, 2019. 